ПАТОГЕНЕТИЧЕСКИЕ ФАКТОРЫ ПАТОЗООСПЕРМИИ И НАРУШЕНИЯ АНТИОКСИДАНТНОЙ АКТИВНОСТИ ЭЯКУЛЯТА У МОЛОДЫХ МУЖЧИН С ПОСТПУБЕРТАТНЫМ ВИСЦЕРАЛЬН ОЖИРЕНИЕМ И НЕОТЯГОЩЕННЫМ АНДРОЛОГИЧЕСКИМ АНАМНЕЗОМ

(с) Р.В. Роживанов* , Д.Н. Бобков, Д.Г. Курбатов

ФГБУ «Национальный медицинский исследовательский центр эндокринологии» Минздрава России, Москва, Россия

Обоснование. Учитывая негативное влияние висцерального ожирения на сперматогенез, актуально исследование его патогенетических факторов.

Цель. Выявление патогенетических факторов патозооспермии и нарушения антиоксидантной активности (АОА) эякулята у пациентов с постпубертатным висцеральным ожирением и неотягощенным андрологическим анамнезом. Memoды. В сплошное одномоментное исследование были включены 47 мужчин с постпубертатным алиментарным висцеральным ожирением в возрасте до 30 лет. Проводилась оценка длины окружности талии (ОT), липидного спектра крови, уровней глюкозы и инсулина, АОА эякулята, его электронно-микроскопическое исследование. Статистически значимыми считали различия между группами при $\mathrm{p}<0,05$.

Результаты. Уровни липопротеидов низкой плотности (ЛПНП) и триглицеридов (ТГ) у пациентов с патозооспермией были статистически значимо выше наблюдаемых при нормозооспермии. Были выявлены статистически значимые отрицательные корреляция между АОА эякулята и уровнями ЛПНП ( $n=47 ; r=-0,310 ; p=0,033)$, а также ТГ ( $n=47 ; r=-0,366$; $\mathrm{p}=0,011)$. Была выявлена статистически значимая положительная корреляция между АОА эякулята и содержанием в эякуляте нормальных форм сперматозоидов ( $n=47 ; \mathrm{r}=0,343 ; \mathrm{p}=0,017)$.

Заключение. У молодых мужчин с постпубертатным висцеральным ожирением и неотягощенным андрологическим анамнезом нарушения липидного спектра крови могут являться патогенетическими факторами патозооспермии. Однако действие дислипидемии на сперматогенез может быть опосредовано через снижение АОА эякулята вследствие развития оксидативного стресса.

КЛЮЧЕВЫЕ СЛОВА: ожирение; сперматогенез; оксидативный стресс.

\title{
PATHOGENETIC FACTORS OF DISORDERS IN SPERMATOGENESIS AND ANTIOXIDATIVE ACTIVITY OF AN EJACULATE IN YOUNG MEN WITH POST PUBERTAL VISCERAL OBESITY AND NORMAL ANDROLOGICAL ANAMNESIS
}

\author{
(c) Roman V. Rozhivanov*, Daniil N. Bobkov, Dmitriy G. Kurbatov
}

Endocrinology Research Centre, Moscow, Russia

BACKGRAUND: Considering the negative influence of a visceral obesity on spermatogenesis, it is important to investigate its pathogenetic factors.

AIMS: To reveal the pathogenetic factors of disorders in spermatogenesis and antioxidative activity of an ejaculate in young men with post pubertal visceral obesity and normal andrological anamnesis.

MATERIALS AND METHODS: 47 men under 30 years with post pubertal and nutritional visceral obesity have been included into one-stage research. The length of circle of waist, lipid blood spectrum, blood levels of glucose and insulin, antioxidant activity and electronic-microscopic analysis of an ejaculate have been examined. Differences were considered statistically significant with $\mathrm{p}<0,05$.

RESULTS: The LDL and triglyceride levels in patients with disorders in spermatogenesis were significantly higher than considered in men with normozoospermia. Correlations between antioxidative activity of ejaculate and levels of $L D L$ ( $n=47$, $r=-0,310 ; p=0,033)$, triglyceride $(n=47, r=-0,366 ; p=0,011)$ and the number of normal spermatozoons in ejaculate ( $n=47$, $r=0,343 ; p=0,017$ ) have been revealed.

CONCLUSIONS: The dyslipidemia in young men with post pubertal visceral obesity and normal andrological anamnesis can be considered as pathogenetic factors of disorders in spermatogenesis. Nevertheless the dyslipidemia can influence spermatogenesis through oxidative stress.

KEYWORDS: obesity; spermatogenesis; oxidative stress. 


\section{ОБОСНОВАНИЕ}

В наших ранних работах была установлена большая распространенность патозооспермии у мужчин с висцеральным ожирением по сравнению со здоровыми людьми даже с учетом отсутствия пациентов с отягощенным андрологическим анамнезом [1, 2]. Одним из типичных патогенетических факторов патозооспермии при ожирении является гипогонадизм [3]. Однако другие патогенетические факторы патозооспермии и нарушения антиоксидантной активности (АОА - комплекс антиоксидантов спермоплазмы, угнетающий радикалообразование на различных фазах цепной реакции свободнорадикального окисления и защищающий сперматозоиды от повреждений) эякулята у таких пациентов остаются неустановленными.

\section{ЦЕЛЬ}

Выявление патогенетических факторов патозооспермии и нарушения АОА эякулята у пациентов с постпубертатным висцеральным ожирением и неотягощенным андрологическим анамнезом.

\section{МЕТОДЫ}

\section{Дизайн исследования}

Сплошное одномоментное исследование.

\section{Критерии соответствия}

В исследование включались совершеннолетние пациенты мужского пола с постпубертатным алиментарным висцеральным (окружность талии (ОТ) от 98 см) ожирением в возрасте до 30 лет включительно. Критериями исключения являлись: индекс массы тела (ИМТ) $\geq 35$ кг/м², объем любого из яичек менее 15 мл, нарушения кариотипа, задержка полового развития, наличие в анамнезе крипторхизма, варикоцеле, воспалительных заболеваний, опухолей, травм или хирургических вмешательств на половых органах и области головного мозга, включая гипофиз, криптозооспермия, азооспермия, бактериоспермия, лейкоспермия, урогенитальные инфекции, носительство антиспермальных антител, сахарный диабет, гипогонадизм, гипотиреоз, гиперпролактинемия, гиперкортицизм.

\section{Условия проведения}

Пациенты, проходившие обследование в отделении андрологии и урологии ФГБУ «НМИЦ эндокринологии» Минздрава России.

\section{Продолжительность исследования}

Исследование одномоментное, данные собирались однократно.

\section{Описание медицинского вмешательства}

Осуществлялся забор крови в пробирки типа «вакутейнер» в утреннее время натощак из локтевой вены, а также эякулята в стерильные контейнеры путем мастурбации (половое воздержание 3-5 сут).

\section{Основной исход исследования}

Основным исходом исследования является получение данных о патогенетических факторах патозооспер- мии и нарушениях АОА эякулята у молодых мужчин с висцеральным ожирением.

\section{Дополнительные исходы исследования}

Дополнительным исходом исследования является установление взаимосвязи выявленных патогенетических факторов с количественными показателями сперматогенеза у молодых мужчин с висцеральным ожирением.

\section{Анализ в подгруппах}

Включенные в исследование пациенты были разделены на 2 группы: 1-я группа - мужчины с нормозооспермией и нормальной АОА эякулята, 2-я группа - мужчины с патозооспермией и нарушенной АOA эякулята.

\section{Методы регистрации исходов}

У всех пациентов были определены ИМТ и ОТ. Концентрацию биохимических показателей сыворотки крови - холестерина (XC), холестерина липопротеидов низкой плотности (ЛПНП), холестерина липопротеидов высокой плотности (ЛПВП), триглицеридов (ТГ), глюкозы определяли на биохимическом анализаторе HITACHI (Biohringer Mannheim, Япония). Уровни инсулина определяли на автоматическом хемилюминесцентном анализаторе Autodelfia (Wallac, Финляндия). На основании уровней глюкозы и инсулина рассчитывался индекс НОМА. Оценка спермограмм осуществлялась в соответствии с рекомендациями ВО3, 2010 г. путем световой микроскопии с помощью микроскопа Olimpus 41 CX (Япония) и камеры Маклера того же производителя [4]. Учитывая, что на параметры спермограммы может влиять множество различных факторов, ее анализировали двукратно (ориентировались на лучший результат). Кроме того, с целью оценки содержания сперматозоидов с интактными головками и отсутствием патологических изменений (норма >4\%), сперматозоидов с недостаточно конденсированным хроматином (норма <30\%), а также оценки патологических изменений головки, шейки, акросомы и жгутика сперматозоидов проводилось электронно-микроскопическое исследование эякулята (ЭМИС). Эякулят после разжижения фиксировали 2,5\% раствором глутарового альдегида на 0,1M какодилатном буфере (рН 7,2-7,4) и 1\% р-ром осмиевой кислоты и заливали в эпоксидную смолу. Ультратонкие срезы получали на ультрамикротоме Reichert III и просматривали в электронном микроскопе Hitachi 700 [5-7]. AOA эякулята (норма 1,5-3,2 мМ-экв) определялась с использованием многофункционального потенциометрического анализатора «МПА-1» (НПВП «Ива», г. Екатеринбург) и медиаторной системы, содержащей $\mathrm{K}_{3}\left[\mathrm{Fe}(\mathrm{CN})_{6}\right] / \mathrm{K}_{4}\left[\mathrm{Fe}(\mathrm{CN})_{6}\right]$ в соотношении 0,01M/0,0001M. Аликвота эякулята 0,2 мл, буферный раствор с медиаторной системой - 1 мл [8].

\section{Этическая экспертиза}

Протокол №1 от 01.02.2017 г. заседания ученого совета ФГБУ «Эндокринологический научный центр» Минздрава России. 
Статистический анализ

Принципы расчета размера выборки: размер выборки предварительно не рассчитывался.

Методы статистического анализа данных. Статистическая обработка полученных данных была проведена с использованием пакета прикладных программ Statistica (StatSoft Inc. США, версия 8.0). Сравнение двух независимых групп осуществлялось непараметрическим методом с использованием U-критерия МаннаУитни. Анализ связи (корреляции) двух количественных признаков осуществлялся непараметрическим методом ранговой корреляции по Спирмену. Количественные данные представлены в виде медиан и границ интерквартильного интервала. Статистически значимыми считали различия при $\mathrm{p}<0,05$.

\section{РЕЗУЛЬТАТЫ}

Объекты (участники) исследования

В сплошное одномоментное исследование были включены 47 совершеннолетних пациентов мужского пола с постпубертатным алиментарным висцеральным (ОТ >98 см) ожирением в возрасте до 30 лет.

\section{Основные результаты исследования}

Показатели качества сперматогенеза и АOA эякулята мужчин 2-й группы были статистически значимо хуже таковых у мужчин 1-й группы, что было обусловлено межгрупповым делением (табл. 1). При этом для пациентов 2-й группы была характерна статистически значимо большая выраженность ожирения, нежели

Таблица 1. Результаты обследования мужчин

\begin{tabular}{|c|c|c|c|}
\hline Параметр & $\begin{array}{c}\text { 1-я группа } \\
n=10\end{array}$ & $\begin{array}{c}\text { 2-я группа } \\
n=37\end{array}$ & $\mathbf{p}^{*}$ \\
\hline Возраст, лет & $27[26 ; 29]$ & $29[27 ; 30]$ & 0,091 \\
\hline ИМТ, $\mathrm{kг} / \mathrm{M}^{2}$ & $26,9[26,5 ; 27,4]$ & $28,7[27,8 ; 30,3]$ & 0,003 \\
\hline OT, $\mathrm{CM}$ & $99[98 ; 100]$ & $101[99 ; 107]$ & 0,052 \\
\hline Холестерин, ммоль/л & $5,4[4,8 ; 5,7]$ & $5,6[5,4 ; 6,0]$ & 0,107 \\
\hline ЛПНП, ммоль/л & $3,2[2,6 ; 3,5]$ & $3,7[3,3 ; 4,5]$ & 0,020 \\
\hline лПВП, ммоль/л & $1,3[1,2 ; 1,4]$ & $1,0[0,9 ; 1,1]$ & 0,007 \\
\hline ТГ, ммоль/л & $1,4[1,0 ; 1,6]$ & $1,9[1,6 ; 2,3]$ & 0,014 \\
\hline Индекс НОМА & $2,4[2,2 ; 2,6]$ & $2,5[2,2 ; 3,5]$ & 0,316 \\
\hline Срок воздержания, дни & $3[3 ; 4]$ & $4[3 ; 5]$ & 0,968 \\
\hline Объем эякулята, мл & $4,1[2,1 ; 5,0]$ & $3,0[2,5 ; 4,0]$ & 0,420 \\
\hline Вязкость эякулята, мм & $4[3 ; 15]$ & $15[3 ; 15]$ & 0,336 \\
\hline $\mathrm{pH}$ & $7,7[7,6 ; 7,9]$ & $7,7[7,6 ; 7,8]$ & 0,886 \\
\hline Время разжижения, мин & $20[20 ; 20]$ & $20[20 ; 25]$ & 0,316 \\
\hline Кол-во сперматозоидов в 1 мл эякулята, млн & $76[43 ; 107]$ & $31[21 ; 46]$ & 0,003 \\
\hline Живые сперматозоиды, \% & $93[90 ; 96]$ & $90[84 ; 92]$ & 0,031 \\
\hline Подвижность сперматозоидов a+b, \% & $60[57 ; 60]$ & $48[25 ; 62]$ & 0,029 \\
\hline Подвижность сперматозоидов а, \% & $45[40 ; 48]$ & $10[1 ; 22]$ & $<0,001$ \\
\hline Подвижность сперматозоидов b, \% & $14[12 ; 16]$ & $27[20 ; 40]$ & 0,003 \\
\hline Подвижность сперматозоидов с, \% & $7[5 ; 12]$ & $10[6 ; 11]$ & 0,612 \\
\hline Подвижность сперматозоидов d, \% & $31[26 ; 36]$ & $39[24 ; 59]$ & 0,104 \\
\hline Нормальные формы сперматозоидов, \% & $10[6 ; 12]$ & $4[2 ; 8]$ & 0,001 \\
\hline Дефекты головки сперматозоидов, \% & $81[79 ; 84]$ & $87[84 ; 89]$ & $<0,001$ \\
\hline Дефекты шейки сперматозоидов, \% & $16[15 ; 18]$ & $17[15 ; 19]$ & 0,612 \\
\hline Дефекты акросомы сперматозоидов, \% & $6[6 ; 8]$ & $8[5 ; 11]$ & 0,236 \\
\hline Дефекты жгутиков сперматозоидов, \% & $7[3 ; 8]$ & $5[3 ; 7]$ & 0,735 \\
\hline Многоядерные сперматозоиды, \% & $0[0 ; 2]$ & $0[0 ; 1]$ & 0,906 \\
\hline Деградировавшие сперматозоиды, \% & $1[0 ; 2]$ & $1[0 ; 2]$ & 0,979 \\
\hline $\begin{array}{l}\text { Сперматозоиды с недостаточно } \\
\text { конденсированным хроматином, \% }\end{array}$ & $22[21 ; 27]$ & $45[38 ; 56]$ & $<0,001$ \\
\hline Антиоксидантная активность эякулята, мМ-экв & $2,3[2,1 ; 2,4]$ & $0,9[0,8 ; 1,1]$ & $<0,001$ \\
\hline
\end{tabular}

* U-критерий Манна-Уитни; различия статистически значимы при p<0,05. 
у пациентов 1-й группы. Кроме того, уровни ЛПНП и ТГ у пациентов 2-й группы были статистически значимо выше наблюдаемых в 1-й группе, а уровни ЛПВП, напротив, статистически значимо ниже, при отсутствии статистически значимых различий в уровне ХС и индексе HOMA.

\section{Дополнительные результаты исследования}

При проведении корреляционного анализа не было выявлено статистически значимых корреляций между показателями сперматогенеза и липидного спектра крови, но были выявлены статистически значимые отрицательные корреляция между AOA эякулята и уровнями ЛПНП $(n=47 ; r=-0,310 ; p=0,033)$, а также ТГ ( $n=47 ; r=-0,366 ; p=0,011)$. При этом были выявлены статистически значимая положительная корреляция между АОА эякулята и содержанием в эякуляте нормальных форм сперматозоидов $(n=47 ; \quad r=0,343 ; p=0,017)$, а также статистически значимая отрицательная корреляция между АОА эякулята и содержанием в эякуляте сперматозоидов с недостаточно конденсированным хроматином $(n=47 ; r=-0,858 ; p=<0,001)$.

Нежелательные явления

Нежелательные явления отсутствовали.

\section{ОБСУЖДЕНИЕ}

\section{Резюме основного результата исследования}

Нарушения липидного спектра крови могут являться патогенетическим фактором патозооспермии при висцеральном ожирении. Однако действие дислипидемии на сперматогенез может быть опосредовано через снижение АOА эякулята.

\section{Обсуждение основного результата исследования}

Повышенный уровень ЛПНП и ТГ у пациентов с патозооспермией может свидетельствовать о роли этих патогенетических факторов в развитии нарушений сперматогенеза при ожирении. Тем не менее нами не было выявлено корреляций между ЛПнП или ТГ и количественными показателями сперматогенеза. Это может объясняться тем, что дислипидемия не действует на сперматогенез напрямую, а оказывает свое негативное воздействие через развитие оксидативного стресса. По данным зарубежных исследований, дислипидемия in vitro сопровождалась развитием оксидативного стресса [9], а в клиническом исследовании приводила к снижению числа нормальных сперматозоидов [10]. В нашем исследовании уровни ЛПНП и ТГ отрицательно коррелировали с AOA эякулята, в то время как AOA эякулята положительно коррелировала с содержанием нормальных сперматозоидов, что подтверждает патогенез действия дислипидемии при патозооспермии через развитие оксидативного стресса. На роль оксидативного стресса в развитии нарушений сперматогенеза указывают и работы других авторов [11-13]. В отношении показателей углеводного обмена нами не было установлено их влияния на сперматогенез. В других работах отмечается негативное влияние гиперинсулинемии и инсулинорезистентности на показатели сперматогенеза и развитие оксидативного стресса [14-16]. Отсутствие этого влияния в нашей работе может объясняться молодым возрастом пациентов и отсутствием у них нарушений углеводного обмена.

Ограничения исследования

Формирование выборки проводилось из пациентов, наблюдавшихся в крупном федеральном медицинском центре, следовательно, данные о состоянии сперматогенеза в общей популяции мужчин могут отличаться от полученных в исследовании.

\section{ЗАКЛЮЧЕНИЕ}

У молодых мужчин с постпубертатным висцеральным ожирением и неотягощенным андрологическим анамнезом нарушения липидного спектра крови могут являться патогенетическими факторами патозооспермии. Однако действие дислипидемии на сперматогенез может быть опосредовано через снижение АОА эякулята вследствие развития оксидативного стресса.

\section{ДОПОЛНИТЕЛЬНАЯ ИНФОРМАЦИЯ}

Источник финансирования. Исследование выполнено в рамках государственного задания - «Сигнальные молекулы адипоцитов: геномные и постгеномные механизмы реализации физиологической и патогенетической функций жировой ткани при эндокринопатиях» Номер государственного учета НИОКТР АААА-А17-117012610112.

Конфликт интересов. Авторы декларируют отсутствие явных и потенциальных конфликтов интересов, связанных с публикацией настоящей статьи.

Информация о вкладе каждого автора. Роживанов Р.В. - разработка концепции исследования, сбор научного материала, статистическая обработка данных, написание текста; Бобков Д.Н. - сбор научного материала, написание текста; Курбатов Д.Г. - разработка концепции исследования, редактирование текста.

\section{СПИСОК ЛИТЕРАТУРЫ | REFERENCES}

1. Роживанов Р.В., Курбатов Д.Г. Структура патозооспермии у молодых мужчин с постпубертатным висцеральным ожирением и неотягощенным андрологическим анамнезом // Ожирение и метаболизм. - 2017. - Т. 14 №4. - C. 32-37. [Rozhivanov RV, Kurbatov DG. The structure of pathozoospermia in young men with post-pubertal visceral obesity and a non-aggravated andrologic anamnesis // Obesity and metabolism. 2017;(14)4:32-37. (in Russ).] doi: https://doi.org/10.14341/omet2017432-37
2. Роживанов Р.В., Курбатов Д.Г. Состояние сперматогенеза и антиоксидантной активности эякулята у молодых мужчин с постпубертатным висцеральным ожирением и неотягощенным андрологическим анамнезом // Ожирение и метаболизм. - 2018. - T. 15. - №4. - C. 38-43. [Rozhivanov RV, Kurbatov DG. The spermatogenesis and antioxidative activity of an ejaculate in young men with post pubertal visceral obesity and normal andrological anamnesis // Obesity and metabolism. 2018;(15)4:38-43. doi: https://doi.org/10.14341/omet9609 
3. Витязева И.И., Алташина М.В., Трошина Е.А. Влияние нарушений жирового обмена на фертильность мужчин репродуктивного возраста и эффективность программ ЭКО // Проблемы Эндокринологии. - 2014. - Т. 60. №5. - C. 34-42. [Vityazeva II, Altashina MV, Troshina EA The influence of disordered fat metabolism on male fertility at the reproductive age and the effectiveness of the ECF programs // Problems of Endocrinology. 2014;(60)5:34-42. (in Russ).] doi: https://doi.org/10.14341/probl201460534-42

4. Всемирная организация здравоохранения, Департамент репродуктивного здоровья и научных исследований Руководство ВОЗ по исследованию и обработке эякулята человека. Пятое издание. - М.: КАПИТАЛ ПРИНТ, 2012. Доступно по: https://www.who.int/reproductivehealth/publications/ infertility/9789241547789/ru/

5. Хаят Ш., Брагина Е.Е., Курило Л.Ф. Ультраструктурное исследование сперматозоидов у пациентов с астенозооспермией // Андрология и генитальная хирургия. - 2012. - Т.13. - №4. C. 54-61. [Khayat SS, Bragina EE, Kurilo LF. Ultrastructural investigation of human sperm from asthenozoospermic men. Andrology and genital surgery journal. 2012;13(4):54-61. (In Russ).]

6. Брагина Е.Е., Бочарова Е. Н. Количественное электронномикроскопическое исследование сперматозоидов при диагностике мужского бесплодия // Андрология и генитальная хирургия. - 2014. - Т.15. - №1. - С. 54-63. [Bragina YY, Bocharova YN. Quantitative electron microscopic examination of sperm for male infertility diagnosis. Andrology and genital surgery journal. 2014;15(1):41-50. (In Russ).]

7. Брагина Е.Е., Замятнина В.А., Бочарова Е.Н., и др. Количественное ультраструктурное исследование хроматина сперматозоидов при нарушении фертильности // Андрология и генитальная хирургия. 2009;1:44-49. [Bragina EE, Zamyatnina VA, Bocharova EN, et al. Quantitative ultrastructural research research of spermatozoon from patients with fertility infringement. Andrology and genital surgery journal. 2009;(1):44-49. (In Russ).]. eLIBRARY ID: 12516321

8. Герасимова Е.Л. Потенциометрия в исследовании антиоксидантной активности биологических объектов / Сборник тезисов Съезда аналитиков России и Школы молодых ученых
«Аналитическая химия - новые методы и возможности»: Москва, 26-30 апреля 2010 г. - М.: ГЕОХИ РАН, 2010. - C.79. [Gerasimova EL. Potentiometry in the study of the antioxidant activity of biological objects. In: Proceedings of the Congress of Analysts of Russia and the School of Young Scientists «Analytical Chemistry - New Methods and Opportunities»; Moscow, April 26-30 2010. Moscow: GEOCHI RAS; 2010. P.79 (In Russ.)

9. Koppers AJ, Garg ML, Aitken RJ. Stimulation of mitochondrial reactive oxygen species production by unesterified, unsaturated fatty acids in defective human spermatozoa. Free Radic Bio/Med. 2010;48(1):112-119. doi: https://doi.org/10.1016/j.freeradbiomed.2009.10.033

10. Schisterman EF, Mumford SL, Chen Z, et al. Lipid concentrations and semen quality: the LIFE study. Andrology. 2014;2(3):408-415. doi: https://doi.org/10.1111/j.2047-2927.2014.00198.x

11. Божедомов В.А., Громенко Д.С., Ушакова И.В., и др. Причины оксидативного стресса сперматозоидов // Проблемы репродукции. - 2008. - T. 14. - №6. - C. 67-73. [Bozhedomov VA, Gromenko DS, Ushakova IV, et al. Prichiny oksidativnogo stressa spermatozoidov. Problemy reproduktsii. 2008;(14)6:67-73. (In Russ).].

12. Shiraishi K, Takihara H, Matsuyama H. Elevated scrotal temperature, but not varicocele grade, reflects testicular oxidative stress-mediated apoptosis. World J Urol. 2010;28(3):359-364. doi: https://doi.org/10.1007/s00345-009-0462-5

13. Samavat J, Natali I, Degl'Innocenti S, et al. Acrosome reaction is impaired in spermatozoa of obese men: a preliminary study. Fertil Steril. 2014;102(5):1274-1281.e2. doi: https://doi.org/10.1016/j.fertnstert.2014.07.1248

14. Lampiao F, du Plessis SS. Insulin and leptin enhance human sperm motility, acrosome reaction and nitric oxide production. Asian J Androl. 2008;10(5):799-807. doi: https://doi.org/10.1111/j.1745-7262.2008.00421.x

15. La Vignera S, Condorelli R, Vicari E, et al. Diabetes Mellitus and Sperm Parameters. J Androl. 2012;33(2):145-153. doi: https://doi.org/10.2164/jandrol.111.013193

16. Leisegang K, Bouic PJ, Menkveld R, Henkel RR. Obesity is associated with increased seminal insulin and leptin alongside reduced fertility parameters in a controlled male cohort. Reprod Biol Endocrinol. 2014;12(1):34. doi: https://doi.org/10.1186/1477-7827-12-34

ИНФОРМАЦИЯ ОБ АВТОРАХ [AUTHORS INFO]:

*Роживанов Роман Викторович, д.м.н. [Roman V. Rozhivanov, MD, PhD]; адрес: Россия, 117036, Москва, ул. Дм. Ульянова, д. 11 [address: 11 Dm. Ulyanova street, 117036 Moscow, Russia]; ORCID: http://orcid.org/0000-0002-5386-4289; eLibrary SPIN: 8052-3310; e-mail: rrozhivanov@mail.ru

Бобков Даниил Николаевич, аспирант [Daniil N. Bobkov, MD, postgraduate student]; ORCID: http://orcid.org/0000-0002-7145-8954; eLibrary SPIN: 5311-5050; e-mail: danil-0000@mail.ru Курбатов Дмитрий Геннадьевич, д.м.н., професcop [Dmitriy G. Kurbatov, MD, PhD, Professor]; ORCID: http://orcid.org/0000-0002-5660-6760; eLibrary SPIN: 3675-0978; e-mail: kurbatov.d@mail.ru

*Авторы, ответственные за переписку.

\section{ЦИТИРОВАТЬ:}

Роживанов Р.В., Бобков Д.Н., Курбатов Д.Г. Патогенетические факторы патозооспермии и нарушения антиоксидантной активности эякулята у молодых мужчин с постпубертатным висцеральным ожирением и неотягощенным андрологическим анамнезом // Ожирение и метаболизм. — 2019. — Т.16. — №3. — C.76-80. doi: https://doi.org/10.14341/omet10054

\section{TO CITE THIS ARTICLE:}

Rozhivanov RV, Bobkov DN, Kurbatov DG. Pathogenetic factors of disorders in spermatogenesis and antioxidative activity of an ejaculate in young men with post pubertal visceral obesity and normal andrological anamnesis. Obesity and metabolism. 2019;16(3):76-80. doi: https://doi.org/10.14341/omet10054 